\title{
Large contribution of virtual Delbrück scattering to the emission of photons by relativistic nuclei in nucleus-nucleus and electron-nucleus collisions
}

\author{
I.F. Ginzburg, ${ }^{1, a}$, U.D. Jentschura ${ }^{2,3, b}$, V.G. Serbo ${ }^{4,2, c}$ \\ 1 Sobolev Institute of Mathematics, pr. Koptyuga 4, 630090 Novosibirsk, Russia \\ 2 Institut für Theoretische Physik, Philosophenweg 16, 69120 Heidelberg, Germany \\ 3 Max-Planck-Institut für Kernphysik, Postfach 103980, 69029 Heidelberg, Germany \\ ${ }^{4}$ Novosibirsk State University, Pirogova 2, 630090 Novosibirsk, Russia
}

Received: 2 October 2007 / Revised version: 20 November 2007 /

Published online: 1 February 2008 - (C) Springer-Verlag / Società Italiana di Fisica 2008

\begin{abstract}
Delbrück scattering is the elastic scattering of a photon in the Coulomb field of a nucleus via a virtual electron loop. The contribution of this virtual subprocess to the emission of a photon in the collision of ultra-relativistic nuclei, $Z_{1} Z_{2} \rightarrow Z_{1} Z_{2} \gamma$, is considered. We identify the incoming virtual photon as being generated by one of the relativistic nuclei involved in the binary collision and the scattered photon as being emitted in the process. The energy and angular distributions of the photons are calculated. The discussed process has no infrared divergence. The total cross section obtained is 14 barn for $\mathrm{Au}-\mathrm{Au}$ collisions at the RHIC collider and 50 barn for $\mathrm{Pb}-\mathrm{Pb}$ collisions at the LHC collider. These cross sections are considerably larger than those for ordinary tree-level nuclear bremsstrahlung in the considered photon energy range, $m_{e} \ll E_{\gamma} \ll m_{e} \gamma$, where $\gamma$ is the Lorentz factor of the nucleus. Finally, photon emission in electron-nucleus collisions, $e Z \rightarrow e Z \gamma$, is discussed in the context of the eRHIC option.
\end{abstract}

\section{Introduction and main results}

Recently, electromagnetic processes in ultra-relativistic nuclear collisions were discussed in numerous papers (see the review [1] and references therein), which is connected mainly with the operation of the RHIC collider and the future LHC lead-lead option. For these colliders the charge numbers of the nuclei $Z_{1}=Z_{2} \equiv Z$ and their Lorentz factors $\gamma_{1}=\gamma_{2} \equiv \gamma$ are given in Table 1 , which is cited here from [2].

Strictly speaking, only a few electromagnetic processes with the production of leptons or photons are related to fundamental physics. Nevertheless, many of them are of imminent importance for two reasons: they are either "dangerous," e.g. in terms of possible beam losses, or they are by contrast quite useful for experiments at the RHIC and LHC colliders. These statements may be illustrated by two examples.

(i) $e^{+} e^{-}$pair production, $Z_{1} Z_{2} \rightarrow Z_{1} Z_{2} e^{+} e^{-}$. In a typical ultra-relativistic collision, the number of the produced electrons is so huge that some of them can be captured by nuclei, which immediately leads to loss of these nuclei from the beam. This capture process is an essential

\footnotetext{
${ }^{\text {a }}$ e-mail: ginzburg@math.nsc.ru

b e-mail: Ulrich.Jentschura@mpi-nd.mpg.de

c e-mail: serbo@math.nsc.ru
}

limitation for the life time of the beam and determines the maximal luminosity of a machine [1].

(ii) Coherent bremsstrahlung. For the usual bremsstrahlung the number of photons emitted in a single collision of bunches is proportional to the number of particles in the first and second bunches: $\mathrm{d} N_{\gamma} \propto N_{1} N_{2} \mathrm{~d} E_{\gamma} / E_{\gamma}$. But when the photon energy decreases, the coherence length becomes comparable to the length of the second bunch, and radiation is caused by the interaction of a nucleus $Z_{1}$ with the second bunch as a whole, but not with each nucleus $Z_{2}$ separately, i.e. coherent bremsstrahlung is an emission of photons by particles of one bunch in the collective electromagnetic field of the oncoming bunch. In this case $\mathrm{d} N_{\gamma}$ becomes proportional to the number of particles in the first bunch and to the squared number of particles in the second bunch: $\mathrm{d} N_{\gamma} \propto N_{1} N_{2}^{2} \mathrm{~d} E_{\gamma} / E_{\gamma}$. As a result, the number of the produced photons at RHIC becomes so huge in the infrared region that this process can be used for monitoring beam collisions $[3,4]$.

In general, these considerations imply that various electromagnetic processes have to be estimated (their cross sections and distributions) in order not to miss some interesting or potentially dangerous effects.

Let us consider an emission of photons in an elastic nuclear collision (i.e., without excitation of the nuclei in 
Table 1. Colliders and cross sections for the $Z Z \rightarrow Z Z \gamma$ process via the Delbrück scattering subprocess

\begin{tabular}{lcrc}
\hline Collider & $Z$ & $\gamma$ & $\sigma$ [barn] \\
\hline RHIC, $\mathrm{Au}-\mathrm{Au}$ & 79 & 108 & 14 \\
$\mathrm{LHC}, \mathrm{Pb}-\mathrm{Pb}$ & 82 & 3000 & 50 \\
\hline
\end{tabular}

their final state). The ordinary nuclear bremsstrahlung has been known in detail for many years (see, for example, the review [5]). It is described by the Feynman diagrams of Fig. 1a and b, in which the virtual photon is emitted by either one of the nuclei and then this photon is Compton scattered by the oncoming nucleus [In this as well as in other figures we display not all, but the representative diagrams only. E.g., in Fig. 1a, the diagram with the exchange of photons with momenta $q$ and $k$ have to be added to complete the entire range of diagrams.]

In the present paper, we consider in detail the emission of photons not due to the Compton subprocess, but due to another one - namely, the Delbrück scattering subprocess of Fig. 2. Delbrück scattering is an elastic scattering of a photon in the Coulomb field of a nucleus via a virtual electron loop. To the best of our knowledge, an initial discussion of the importance of the process Fig. 2 for the production of photons in heavy-ion collisions was given in [6]. We noted that the situation is interesting because for heavy nuclei the cross section of Delbrück scattering is known to be greater by one order of magnitude than for Compton scattering of photons with small energies. Therefore, we expect that the photon emission of Fig. 2 due

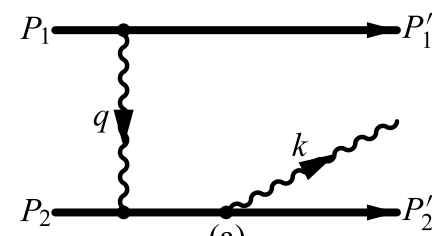

(a)

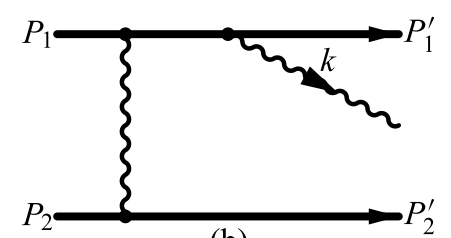

(b)
Fig. 1. Ordinary nuclear bremsstrahlung is the emission of a photon in a nuclear collision via a virtual Compton subprocess

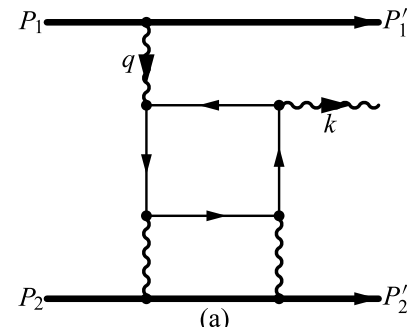

(a)

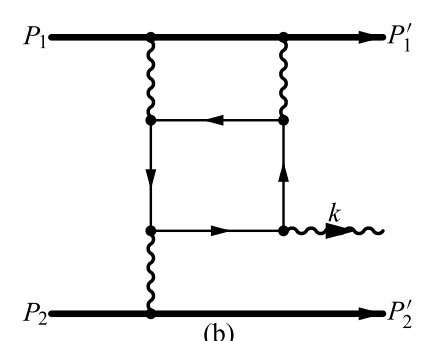

(b)
Fig. 2. Emission of a photon in a nuclear collision via virtual Delbrück scattering in the lowest order of quantum electrodynamics (QED). Bold lines denote nuclei, thin lines denote the electron propagators to Delbrück scattering should be dominant over the ordinary bremsstrahlung of Fig. 1 in a certain range of photon energies.

At first sight, the process of Fig. 2 looks like a typical quantum electrodynamic (QED) loop correction to the Compton scattering and, therefore, should have a small cross section $\sigma \propto \alpha^{7}$, where $\alpha \approx 1 / 137$ is the fine structure constant. However, at second sight, we should add a very large factor $Z^{6} \sim 10^{11}$ (we assume a collision of identical nuclei with $Z=Z_{1}=Z_{2}$ ) and take into account that the natural scale of the cross section is the square of the electron Compton wavelength $\hbar^{2} /\left(m_{e} c\right)^{2}$, where $m_{e}$ is the electron mass, $\hbar$ is the Planck constant and $c$ is the light velocity. And, last but not least, we show below that this cross section has an additional logarithmic enhancement of the order of

$$
L^{2} \gtrsim 100, \quad L=\ln \left(\gamma_{1} \gamma_{2}\right) .
$$

As a result, the discussed cross section is of the order of

$$
\sigma \sim \alpha(Z \alpha)^{6} \frac{\hbar^{2}}{m_{e}^{2} c^{2}} L^{2},
$$

which gives 130 barn for $\mathrm{Pb}-\mathrm{Pb}$ at LHC. A more detailed calculation, as reported below, gives a result that differs from that estimate only by a numerical prefactor approximately equal to 0.4 . The corresponding cross sections are given in Table 1. In particular, for the LHC collider,

$$
\sigma=50 \text { barn } .
$$

Note for comparison, that this cross section is 6 times larger than for the total hadronic/nuclear cross section in $\mathrm{Pb}-\mathrm{Pb}$ collisions, which is roughly 8 barn.

To complete the description, we should mention that there is a numerically not large, but conceptually interesting so-called unitarity correction to the discussed process. It is due to the unitarity requirement for the $S$-matrix and corresponds to the exchange of light-by-light blocks between nuclei (Fig. 3); this correction is analyzed in detail in [7], where it is shown that the unitarity correction can be estimated by the simple expression $\sim-0.5(Z \alpha)^{4}$.

The obtained results can easily be generalized to photon emission in electron-nucleus collisions without excitation of the nucleus, $e Z \rightarrow e Z \gamma$. This consideration is motivated by a project of the eRHIC collider that is now

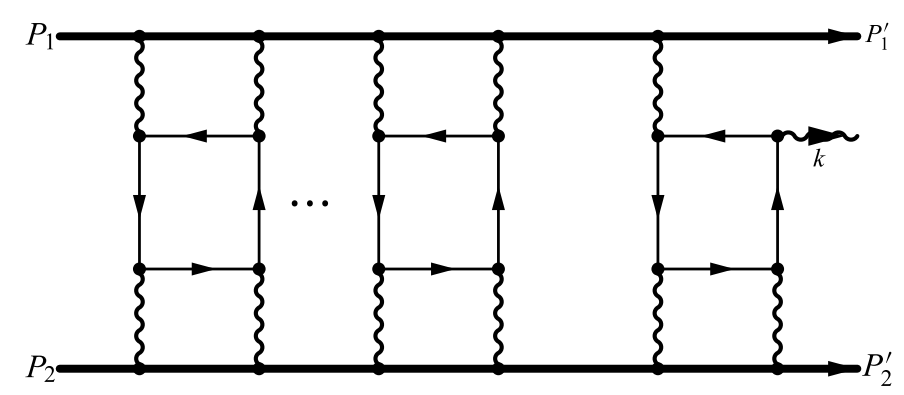

Fig. 3. Feynman diagram for the unitarity correction 
actively discussed as a promising extension of the existing RHIC machine [8]. In particular, the proposal is to build an additional electron ring with the energy $E_{e}=10 \mathrm{GeV}$ and, thus, to create an electron-nucleus collider. Certainly, the emission of photons in the direction of the electron beam is dominated by ordinary bremsstrahlung. By contrast, for the emission in the nuclear beam direction, we find out that the process via Delbrück scattering is dominant in a certain region of the photon energy.

The paper is organized as follows. In Sect. 2, we recall some known properties of Delbrück scattering. In Sect. 3, we calculate the contribution of Delbrück scattering to the cross section of the $Z_{1} Z_{2} \rightarrow Z_{1} Z_{2} \gamma$ process. Section 4 is devoted to a quantitative comparison of our process to other, competing photon emission processes. In Sect. 5 we discuss the case of the electron-nucleus collisions. Final conclusions are given in Sect. 6. A short summary of our main results is presented in [9].

Throughout this text, we use natural units with $\hbar=$ $c=1$ and $\alpha \approx 1 / 137$, and we denote the electron (nucleus) mass by $m \equiv m_{e}(M)$, respectively. The scalar product of two 4 -vectors is $P_{1} \cdot P_{2}=E_{1} E_{2}-\mathbf{P}_{1} \mathbf{P}_{2}$.

\section{Basics of Delbrück scattering}

Delbrück scattering is one of a few fundamental non-linear QED processes that have been studied experimentally. It can be described as a transition, in which the initial real photon fluctuates into a virtual electron-positron pair, interacts with a nucleus and then transforms back to the final photon, i.e. it is elastic scattering of a photon in the Coulomb field of a nucleus via a virtual electron loop. In one-loop order, it is described by the block diagram of Fig. 4. Properties of Delbrück scattering are well known (see, e.g., the review [10], for recent experiments [11] and for numerical results for the Delbrück scattering amplitudes [12]). The total cross section of this process $\sigma_{\mathrm{D}}\left(\omega_{\mathrm{L}}, Z\right)$ depends on the invariant (see Fig. 2 for the identification of $q$ and $P$ )

$$
\omega_{\mathrm{L}}=\frac{q \cdot P}{M},
$$

which is equal to the initial photon energy in the laboratory system (lab-system, denoted by the subscript L). Here the lab-system means the rest frame of the scattering nucleus, in which the 4-momentum of the initial photon takes the form $q=\left(\omega_{\mathrm{L}}, 0,0, \omega_{\mathrm{L}}\right)$, and the 4-momentum of the initial nucleus is $P=(M, 0,0,0)$. This cross section vanishes

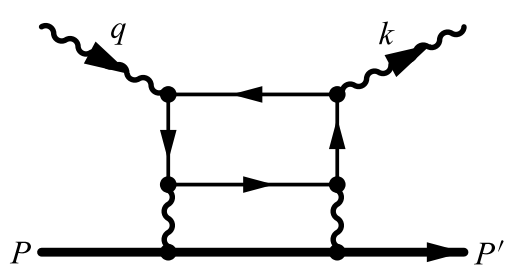

Fig. 4. The block Feynman diagram for Delbrück scattering in the lowest QED order at small energies,

$$
\sigma_{\mathrm{D}}\left(\omega_{\mathrm{L}} \ll m, Z\right) \sim(Z \alpha)^{4} \frac{\alpha^{2}}{m^{2}}\left(\frac{\omega_{\mathrm{L}}}{m}\right)^{4},
$$

and tends to a constant, independent of $\omega_{\mathrm{L}}$, in the limit $\omega_{\mathrm{L}} \gg m$. In the lowest order of QED perturbation theory, this constant is

$$
\sigma_{\mathrm{D}}\left(\omega_{\mathrm{L}} \gg m, Z\right)=\sigma_{\mathrm{D}}^{(0)}(Z)=1.07(Z \alpha)^{4} \frac{\alpha^{2}}{m^{2}} .
$$

For heavy nuclei, the strong-field effects (so-called Coulomb corrections) drastically change this result. These corrections correspond to the exchange of virtual photons between the electron loop and the nucleus. They are of even order in powers of $Z \alpha$ (due to the Furry theorem for the electron loop), i.e. they are proportional to $(Z \alpha)^{2 n}$ and decrease this constant significantly,

$$
\sigma_{\mathrm{D}}^{(0)}(Z) \rightarrow \sigma_{\mathrm{D}}(Z) \equiv \frac{\sigma_{\mathrm{D}}^{(0)}(Z)}{r_{Z}},
$$

where the reduction factor $r_{Z}>1$. For example, for the Delbrück scattering off the $\mathrm{Au}(Z=79)$ and $\mathrm{Pb}(Z=82)$ nuclei, it is $r_{79}=1.7$ and $r_{82}=1.8$, respectively, and the corresponding cross sections read as follows:

$$
\begin{aligned}
& \sigma_{\mathrm{D}}(Z=79)=5.5 \times 10^{-3} \text { barn }, \\
& \sigma_{\mathrm{D}}(Z=82)=6.2 \times 10^{-3} \text { barn } .
\end{aligned}
$$

It should be noted that the cross section for Delbrück scattering off heavy nuclei is considerable larger than that for nuclear Thomson scattering (which is the low-energy limit of Compton scattering),

$$
\sigma_{\mathrm{T}}(Z)=\frac{8 \pi}{3} \frac{Z^{4} \alpha^{2}}{M^{2}},
$$

where $M$ is the mass of nucleus. Indeed, the ratio

$$
\frac{\sigma_{\mathrm{T}}(Z)}{\sigma_{\mathrm{D}}(Z)}=7.83 r_{Z}\left(\frac{m}{\alpha^{2} M}\right)^{2} \approx \frac{1}{30}
$$

for $\mathrm{Au}$ and $\mathrm{Pb}$. For the analysis to be described below, we will also need the differential cross section of Delbrück scattering over the transverse momentum of the final photon $k_{\perp}$ in the region $\omega_{\mathrm{L}} \gg m$. The nucleus can be considered as a point-like particle up to the limit imposed by the nuclear form factor, i.e. up to $k_{\perp} \sim 1 / R$, where $R \approx 1.2 A^{1 / 3} \mathrm{fm}$ is the radius of the nucleus with $A$ the nucleon number (for both $\mathrm{Au}$ and $\mathrm{Pb}$ nuclei, we have $R \approx$ $7 \mathrm{fm}$ and $1 / R \approx 28 \mathrm{MeV})$. In this region, the differential cross section for Delbrück scattering can be written in the form

$$
\mathrm{d} \sigma_{\mathrm{D}}=\alpha^{2}(Z \alpha)^{4} f_{Z}\left(k_{\perp} / m\right) \frac{\mathrm{d} k_{\perp}^{2}}{m_{\perp}^{4}}
$$

with

$$
m_{\perp}=\sqrt{m^{2}+k_{\perp}^{2}} .
$$


The function $f_{Z}\left(k_{\perp} / m\right)$ is of the order of unity at $k_{\perp} / m \lesssim$ 1 (except for the region of very small $k_{\perp} / m \lesssim m / \omega_{\mathrm{L}}$ ), and it is a slowly varying function at larger values of $k_{\perp} / m$. Numerical values of this function can be found from plots and numbers given in $[10,12]$, in particular,

$$
f_{82}\left(k_{\perp} / m=1\right) \approx 0.48, \quad f_{82}\left(k_{\perp} / m \gg 1\right) \approx 1.2 .
$$

As a result, we can conclude that the main contribution to the total cross section at $\omega_{\mathrm{L}} \gg m$ [given in (6)-(8)] comes from the region where the transverse momenta of the final photon are of the order of the electron mass, $k_{\perp} \sim m$.

\section{Delbrück scattering and the $Z_{1} Z_{2} \rightarrow Z_{1} Z_{2} \gamma$ process}

Let us consider, in general terms, the process of a photon emission without any excitation of nuclei in the final state:

$$
Z_{1}\left(P_{1}\right)+Z_{2}\left(P_{2}\right) \rightarrow Z_{1}\left(P_{1}^{\prime}\right)+Z_{2}\left(P_{2}^{\prime}\right)+\gamma(k) .
$$

In this process two nuclei with charges $Z_{1} e$ and $Z_{2} e$ and 4-momenta $P_{1}$ and $P_{2}$ collide with each other and produce a photon with the total 4-momentum $k$. Let $E_{i}\left(\gamma_{i}=\right.$ $E_{i} / M_{i}$ ) and $E_{\gamma}$ be the energy (the Lorentz factor) of the $i$ th nucleus and the photon energy in the collider system, respectively. Here, the collider system means the rest frame of the particle collider, which is not necessarily equal to our "lab-system" (the latter we define to be the rest frame of the scattering nucleus). The collider system, by contrast, coincides with the center-of-mass system for identical colliding nuclei, and in this case $\gamma_{1}=\gamma_{2}=\gamma$.

\subsection{Total cross section}

The contribution of the Delbrück scattering subprocess to the cross section of the process (14) is described in the lowest QED order by the Feynman diagrams displayed in Fig. 2. However, the parameter of the perturbation series $Z \alpha$ is of the order of unity for heavy nuclei. For example, $Z \alpha \approx 0.6$ for the discussed colliders. This means that the other amplitudes $M_{n^{\prime}}^{n}$ (see Fig. 5) with the exchange of $n\left(n^{\prime}\right)$ virtual photons between electron loop and the first (second) nucleus have to be taken into account. Therefore, the whole series in $Z \alpha$ has to be summed to obtain the cross section with sufficient accuracy. Fortunately, there is another small parameter $\eta \equiv 1 / \ln \left(\gamma_{1} \gamma_{2}\right)$, and it will be sufficient to calculate the cross section in the leading logarithmic approximation (LLA), where the omitted terms are of the order of

$$
\eta=\frac{1}{\ln \left(\gamma_{1} \gamma_{2}\right)},
$$

where $\eta=0.1$ for RHIC and 0.06 for LHC.

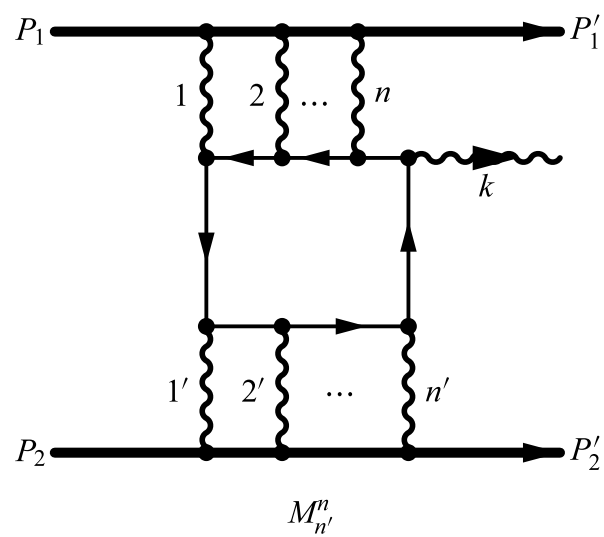

Fig. 5. Amplitude $M_{n^{\prime}}^{n}$ for the emission of a photon in the nuclear collision; here $n\left(n^{\prime}\right)$ is the number of exchange virtual photons between the electron loop and the first (second) nucleus

Let $\mathcal{M}$ be the sum of the amplitudes $M_{n^{\prime}}^{n}$ of Fig. 5. This sum can be presented in the form

$$
\begin{aligned}
\mathcal{M} & =\sum_{n n^{\prime} \geq 1} M_{n^{\prime}}^{n}=M_{1}+\tilde{M}_{1}+M_{2}, \\
M_{1} & =\sum_{n \geq 1} M_{2 n}^{1}, \quad \tilde{M}_{1}=\sum_{n \geq 1} M_{1}^{2 n}, \quad M_{2}=\sum_{n n^{\prime} \geq 2} M_{n^{\prime}}^{n} .
\end{aligned}
$$

The amplitude $M_{1}$ contains a one-photon exchange with the first nucleus and multi-photon exchanges with the second nucleus, whereas the amplitude $\tilde{M}_{1}$ describes a onephoton exchange with the second nucleus and multiphoton exchanges with the first nucleus (Fig. 6). In the last amplitude $M_{2}$, there is no one-photon exchange, it describes a multi-photon exchange between electron loop and both nuclei. According to this classification, we write the total cross section as

$$
\sigma=\sigma_{1}+\tilde{\sigma}_{1}+\sigma_{2}
$$

where

$$
\begin{aligned}
& \mathrm{d} \sigma_{1} \propto\left|M_{1}\right|^{2}, \quad \mathrm{~d} \tilde{\sigma}_{1} \propto\left|\tilde{M}_{1}\right|^{2}, \\
& \mathrm{~d} \sigma_{2} \propto 2 \operatorname{Re}\left(M_{1} \tilde{M}_{1}^{*}+M_{1} M_{2}^{*}+\tilde{M}_{1} M_{2}^{*}\right)+\left|M_{2}\right|^{2} .
\end{aligned}
$$
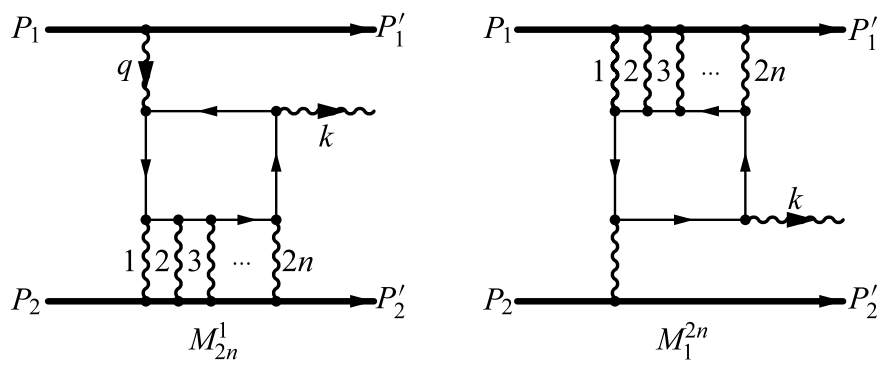

Fig. 6. Amplitudes $M_{2 n}^{1}\left(M_{1}^{2 n}\right)$ with a single exchange photon between the electron loop and the first (second) nucleus 
The integration over the transferred momentum squared $q^{2}$ results in the large Weizsäcker-Williams logarithm $\sim L$ for $\sigma_{1}$, with the same being true for $\tilde{\sigma}_{1}$. The contribution $\sigma_{2}$ does not contain such a logarithm. Therefore, the relative contribution of the $\sigma_{2}$ term is

$$
\frac{\sigma_{2}}{\sigma_{1}} \sim \frac{(Z \alpha)^{2}}{L}<0.04
$$

As a result, with an accuracy of the order of a few percent we can neglect $\sigma_{2}$ in the total cross section and use the equation

$$
\sigma=\sigma_{1}+\tilde{\sigma}_{1}
$$

Let us consider the cross section $\sigma_{1}$, within the 4momentum conventions of Fig. 6 . In the LLA, it can be calculated using the equivalent photon approximation, in which $\mathrm{d} \sigma_{1}$ is expressed via the number of equivalent photons $\mathrm{d} n_{1}$, emitted by the first nuclei, and the cross section for the Delbrück scattering off the second nuclei (see, e.g., [13]):

$$
\mathrm{d} \sigma_{1}=\mathrm{d} n_{1} \sigma_{\mathrm{D}}\left(\omega_{\mathrm{L}}, Z_{2}\right) .
$$

The virtual Delbrück scattering amplitude decreases when the virtuality of the initial photon $Q^{2}=-q^{2}$ becomes larger than $m_{\perp}^{2}$ (here, $q=P_{1}-P_{1}^{\prime}$ is the 4-momentum of the equivalent photon). This means that the main contribution to $\mathrm{d} \sigma_{1}$ is given by photons from the first nucleus with a small virtuality,

$$
Q^{2}=-q^{2}=\mathbf{q}_{\perp}^{2}+\left(\frac{\omega}{\gamma_{1}}\right)^{2} \ll m_{\perp}^{2}
$$

where $\omega=E_{1}-E_{1}^{\prime}$ is the energy of the equivalent photon in the collider system. Therefore, we can neglect the virtuality of this photon in the description of the cross section $\sigma_{\mathrm{D}}\left(\omega_{\mathrm{L}}, Z_{2}\right)$ for the subprocess. From $(22)$, we learn that we can usually assume $\omega \ll m_{\perp} \gamma_{1}$. Because $\omega_{\mathrm{L}}$ can be expressed in terms of Lorentz invariants as

$$
\omega_{\mathrm{L}}=\left(q \cdot P_{2}\right) / M_{2}=2 \omega \gamma_{2},
$$

the most important region for this cross section is [in accordance with (5)-(8) and (22)]

$$
m \ll \omega_{\mathrm{L}}=2 \omega \gamma_{2} \ll m \gamma_{1} \gamma_{2}, \quad k_{\perp} \sim m .
$$

To calculate the spectrum of equivalent photons, we can use (D.4) of [13], neglecting terms proportional to $\omega / E_{1}$, since in our case $\omega \lesssim m \gamma_{1} \ll E_{1}$ :

$$
\mathrm{d} n_{1}\left(\omega, Q^{2}\right)=\frac{Z_{1}^{2} \alpha}{\pi} \frac{\mathrm{d} \omega}{\omega}\left(1-\frac{Q_{\min }^{2}}{Q^{2}}\right) F^{2}\left(Q^{2}\right) \frac{\mathrm{d} Q^{2}}{Q^{2}},
$$

where

$$
Q_{\min }^{2}=\frac{\omega^{2}}{\gamma_{1}^{2}}
$$

and $F\left(Q^{2}\right)$ is the nuclear electromagnetic form factor. The function $F\left(Q^{2}\right)$ is normalized by the "charge conservation" condition $F(0)=1$ but drops very quickly at $Q^{2}$ larger than $1 / R^{2}$. In this section, we assume that $m_{\perp}^{2}$ is considerable smaller than $1 / R^{2}$. This implies, in particular, that $k_{\perp} \ll 1 / R$, and the nucleus is effectively probed in the long-wavelength limit, so that we can put $F\left(Q^{2}\right)=1$ in our calculation. Integrating this number over $Q^{2}$ in the region

$$
Q_{\min }^{2}=\frac{\omega^{2}}{\gamma_{1}^{2}} \leq Q^{2} \lesssim m^{2},
$$

we obtain the spectrum of equivalent photons as

$$
\mathrm{d} n_{1}(\omega)=2 \frac{Z_{1}^{2} \alpha}{\pi} \frac{\mathrm{d} \omega}{\omega} \ln \left(\frac{m \gamma_{1}}{\omega}\right) .
$$

Then integrating the cross section (21) over $\omega$ in the region

$$
\frac{m}{\gamma_{2}} \lesssim \omega \lesssim m \gamma_{1}
$$

we obtain the total cross section $\sigma_{1}$ in the LLA

$$
\begin{aligned}
\sigma_{1} & =\frac{Z_{1}^{2} \alpha}{\pi} \sigma_{\mathrm{D}}\left(Z_{2}\right) L^{2}, \\
L & =\ln \left(\frac{P_{1} \cdot P_{2}}{2 M_{1} M_{2}}\right)=\ln \left(\gamma_{1} \gamma_{2}\right) .
\end{aligned}
$$

Analogously, the cross section $\tilde{\sigma}_{1}$ is

$$
\tilde{\sigma}_{1}=\frac{Z_{2}^{2} \alpha}{\pi} \sigma_{\mathrm{D}}\left(Z_{1}\right) L^{2} .
$$

As a result, the total contribution of virtual Delbrück scattering to the cross section of the process given by (14) is equal to

$$
\sigma=\sigma_{1}+\tilde{\sigma}_{1}=\frac{\alpha}{\pi}\left[Z_{1}^{2} \sigma_{\mathrm{D}}\left(Z_{2}\right)+Z_{2}^{2} \sigma_{\mathrm{D}}\left(Z_{1}\right)\right] L^{2},
$$

where $L$ is given in $(29)$ and $\sigma_{\mathrm{D}}(Z)$ in (7)-(8). In particular, for $\mathrm{Au}-\mathrm{Au}$ collisions at the RHIC collider, the total cross section is $\sigma=14$ barn, and for the $\mathrm{Pb}-\mathrm{Pb}$ collisions at the LHC collider, the total cross section $\sigma=50$ barn (see also Table 1).

\subsection{Energy and angular distribution of final photons}

In the previous subsection, we have considered only the total cross section. Here, we are interested in the angular distribution of the final photons, and therefore, the dependence on $k_{\perp}$ has to be restored. Let us therefore consider the region of not too small transverse momenta,

$$
m \lesssim k_{\perp} \lesssim 1 / R,
$$

which differs from the condition $k_{\perp} \ll 1 / R$ employed in the previous calculation. To obtain the number of equivalent 
photons in this region, we should integrate expression (24) not in the interval (26), but in the larger interval

$$
Q_{\min }^{2}=\left(\omega / \gamma_{1}\right)^{2} \leq Q^{2} \lesssim m_{\perp}^{2},
$$

whose upper limit is $m_{\perp}^{2}$, not $m^{2}$. This leads to

$$
\mathrm{d} n_{1}(\omega)=2 \frac{Z_{1}^{2} \alpha}{\pi} \frac{\mathrm{d} \omega}{\omega} \ln \frac{m_{\perp} \gamma_{1}}{\omega}
$$

Using this expression and the distribution of the Delbrück subprocess [given by (11)], we obtain the differential cross section

$$
\mathrm{d} \sigma_{1}=\frac{2}{\pi} \alpha\left(Z_{1} \alpha\right)^{2}\left(Z_{2} \alpha\right)^{4} \ln \left(\frac{m_{\perp} \gamma_{1}}{\omega}\right) f_{Z_{2}}\left(k_{\perp} / m\right) \frac{\mathrm{d} \omega}{\omega} \frac{\mathrm{d} k_{\perp}^{2}}{m_{\perp}^{4}}
$$

which is valid in the region

$$
\frac{m_{\perp}}{\gamma_{2}} \ll \omega \ll m_{\perp} \gamma_{1}, \quad k_{\perp} \sim m_{\perp} .
$$

To find the energy and angular distribution of the final photons, we should now express the energy of the equivalent photon $\omega$ in this formula in terms of the energy of the final photon $E_{\gamma}$.

In the main region for the cross section $\sigma_{1}$, the energy of the final photon in the rest frame of the second nucleus,

$$
E_{\gamma \mathrm{L}}=\frac{\left(k \cdot P_{2}\right)}{M_{2}}=\omega_{\mathrm{L}}-\frac{k_{\perp}^{2}}{2 M_{2}},
$$

and the longitudinal momentum (in the $z$ direction, as measured in the lab frame L),

$$
k_{z L}=E_{\gamma \mathrm{L}}-\frac{k_{\perp}^{2}}{2 E_{\gamma \mathrm{L}}}
$$

almost coincide with $\omega_{\mathrm{L}}$. Going to the collider frame of reference, we find the energy of the final photon:

$$
E_{\gamma}=\gamma_{2}\left(E_{\gamma \mathrm{L}}-V_{2} k_{z L}\right)=\omega+\frac{k_{\perp}^{2}}{4 \omega}
$$

and its longitudinal momentum

$$
k_{z}=\gamma_{2}\left(k_{z L}-V_{2} \omega_{\mathrm{L}}\right)=\omega-\frac{k_{\perp}^{2}}{4 \omega},
$$

where $\gamma_{2}=1 / \sqrt{1-V_{2}^{2}}$ and $V_{2}$ is the velocity of the second nucleus in the collider system.

For further analysis, it will be useful to split the region (36) into two subregions with small and large energies of the equivalent photon:

$$
\text { subregion } A: \quad \frac{m_{\perp}}{\gamma_{2}} \ll \omega \ll m_{\perp},
$$

and

$$
\text { subregion } B: \quad m_{\perp} \ll \omega \ll m_{\perp} \gamma_{1} \text {. }
$$

Now we can see that the longitudinal momentum of the final photon is positive in region $B$,

$$
k_{z} \approx E_{\gamma} \approx \omega
$$

Therefore, the photon flies along the momentum of the first nucleus $\mathbf{P}_{1}$, and

$\mathrm{d} \sigma_{1}^{(B)}=\frac{2}{\pi^{2}} \alpha\left(Z_{1} \alpha\right)^{2}\left(Z_{2} \alpha\right)^{4} \frac{f_{Z_{2}}\left(k_{\perp} / m\right)}{\left(m^{2}+k_{\perp}^{2}\right)^{2}} \ln \left(\frac{m_{\perp} \gamma_{1}}{E_{\gamma}}\right) \frac{\mathrm{d}^{3} k}{E_{\gamma}}$,

$m_{\perp} \ll E_{\gamma} \ll m_{\perp} \gamma_{1}$.

The contributions of this region to the spectrum of the final photon is

$$
\begin{aligned}
& \mathrm{d} \sigma_{1}^{(B)}=\frac{2}{\pi} Z_{1}^{2} \alpha \sigma_{\mathrm{D}}\left(Z_{2}\right) \ln \left(\frac{m \gamma_{1}}{E_{\gamma}}\right) \frac{\mathrm{d} E_{\gamma}}{E_{\gamma}}, \\
& m \ll E_{\gamma} \ll m \gamma_{1} .
\end{aligned}
$$

On the contrary, the longitudinal momentum of the final photon is negative in subregion $A$ :

$$
k_{z} \approx-E_{\gamma} \approx-\frac{k_{\perp}^{2}}{4 \omega}
$$

Therefore, the photon flies along the momentum of the second nucleus, $\mathbf{P}_{2}=-\mathbf{P}_{1}$, and

$$
\mathrm{d} \sigma_{1}^{(A)}=\frac{2}{\pi^{2}} \alpha\left(Z_{1} \alpha\right)^{2}\left(Z_{2} \alpha\right)^{4} \frac{f_{Z_{2}}\left(k_{\perp} / m\right)}{\left(m^{2}+k_{\perp}^{2}\right)^{2}} \ln \left(\frac{\gamma_{1} E_{\gamma}}{m_{\perp}}\right) \frac{\mathrm{d}^{3} k}{E_{\gamma}}
$$

$m_{\perp} \ll E_{\gamma} \ll m_{\perp} \gamma_{2}$.

The contributions of this region to the spectrum of the final photons is

$$
\begin{aligned}
& \mathrm{d} \sigma_{1}^{(A)}=\frac{2}{\pi} Z_{1}^{2} \alpha \sigma_{\mathrm{D}}\left(Z_{2}\right) \ln \left(\frac{\gamma_{1} E_{\gamma}}{m}\right) \frac{\mathrm{d} E_{\gamma}}{E_{\gamma}}, \\
& m \ll E_{\gamma} \ll m \gamma_{2} .
\end{aligned}
$$

One verifies that reassuringly, after integration over $E_{\gamma}$, the sum of the two expressions (43) and (46) coincides with $\sigma_{1}$ from (29).

The corresponding expressions for $\mathrm{d} \tilde{\sigma}_{1}$ can easily be obtained. For the sum $\mathrm{d} \sigma=\mathrm{d} \sigma_{1}+\mathrm{d} \tilde{\sigma}_{1}$, we present results only for the case of identical nuclei and for the differential over the photon momentum cross section

$$
\begin{aligned}
& \mathrm{d} \sigma=\frac{2}{\pi^{2}} \alpha(Z \alpha)^{6} \frac{f_{Z}\left(k_{\perp} / m\right)}{\left(m^{2}+k_{\perp}^{2}\right)^{2}} L \frac{\mathrm{d}^{3} k}{E_{\gamma}}, \\
& m_{\perp} \ll E_{\gamma} \ll m_{\perp} \gamma
\end{aligned}
$$

and for the spectrum of photons

$$
\mathrm{d} \sigma=\frac{4}{\pi} Z^{2} \alpha \sigma_{\mathrm{D}}(Z) L \frac{\mathrm{d} E_{\gamma}}{E_{\gamma}}, \quad m \ll E_{\gamma} \ll m \gamma .
$$


The typical emission angle of the photon is not very small:

$$
\frac{1}{\gamma} \ll \theta_{\gamma}=\frac{k_{\perp}}{E_{\gamma}} \ll 1 .
$$

It is useful to make two observations.

First remark. The scattering angles of both nuclei in the discussed process are very small but different for specific contributions to $\sigma_{1}$ and $\tilde{\sigma}_{1}$. In the case of $\sigma_{1}$, the first nucleus gets a transverse momentum $P_{1 \perp}^{\prime}=q_{\perp} \ll m$, which is considerably smaller than a typical transverse momentum of the second scattered nucleus $P_{2 \perp}^{\prime} \approx k_{\perp} \sim m$. In the case of $\sigma_{2}$, vice versa, after the scattering process, the first nucleus has a transverse momentum that is considerably larger than that of the second nucleus. That is the reason why the interference of these two contributions is small.

Second remark. The energy distribution of the photons (48) has the form

$$
\mathrm{d} \sigma \propto \frac{\mathrm{d} E_{\gamma}}{E_{\gamma}},
$$

which is typical for the bremsstrahlung spectrum of soft photons and usually leads to an infrared divergence for the total cross section. In our case, this type of distribution is only valid for not too soft photons in the region $m \ll E_{\gamma} \ll m \gamma$. When the photon energy tends to zero, we should take into account that the Delbrück cross section vanishes for soft photons [see (5)]. As a result, the discussed cross section in fact has no infrared divergence.

\section{Comparisons}

\subsection{Comparison to ordinary nuclear bremsstrahlung}

The ordinary photon emission by nuclear bremsstrahlung is described by the block Feynman diagrams of Fig. 1a and $\mathrm{b}$. Let the cross section $\mathrm{d} \sigma_{\mathrm{br}}^{a}$ and $\mathrm{d} \sigma_{\mathrm{br}}^{b}$ correspond to the diagrams of Fig. 1a and b, respectively. The bremsstrahlung cross section is

$$
\mathrm{d} \sigma_{\mathrm{br}}=\mathrm{d} \sigma_{\mathrm{br}}^{a}+\mathrm{d} \sigma_{\mathrm{br}}^{b},
$$

because the interference term is small and can be safely neglected.

Now we can repeat the previous calculations with minor changes. The expression analogous to (21) has the form

$$
\mathrm{d} \sigma_{\mathrm{br}}^{a}=\mathrm{d} n_{1}(\omega) \mathrm{d} \sigma_{\mathrm{C}}\left(\omega, Z_{2}\right),
$$

where $\mathrm{d} n_{1}(\omega)$ is the number of the equivalent photons emitted by the first nucleus, and $\sigma_{\mathrm{C}}$ is the cross section for the Compton scattering of this photon off the second nucleus. In the calculation of $\mathrm{d} n_{1}(\omega)$, we should integrate the expression (24) over $Q^{2}$ up to a limit that is imposed by the decrease of the form factor of the nucleus for large $Q^{2} \gg 1 / R^{2}$. Therefore,

$$
\mathrm{d} n_{1}(\omega)=2 \frac{Z_{1}^{2} \alpha}{\pi} \ln \left(\frac{\gamma_{1}}{\omega R}\right) \frac{\mathrm{d} \omega}{\omega} .
$$

For the Compton cross section, we can use a well-known expression valid for a charged point particle (such an approach gives a good approximation at least in the region of not too energetic photons),

$$
\begin{aligned}
\mathrm{d} \sigma_{\mathrm{C}}\left(\omega, Z_{2}\right) & =\frac{3}{2} \sigma_{\mathrm{T}}\left(Z_{2}\right)\left(x-2 x^{2}+2 x^{3}\right) \frac{\mathrm{d} E_{\gamma}}{E_{\gamma}}, \\
x & =\frac{\omega_{\min }}{\omega}, \quad \omega_{\min }=\frac{E_{\gamma}}{4 \gamma_{2}^{2}},
\end{aligned}
$$

where the nuclear Thomson cross section $\sigma_{\mathrm{T}}(Z)$ is given in (9).

Integrating (52) over $\omega$ from $\omega_{\min }$, we find (the upper limit of this integration can be set to infinity due to the fast convergence of the integral)

$$
\mathrm{d} \sigma_{\mathrm{br}}^{a}=\frac{2}{\pi} Z_{1}^{2} \alpha \sigma_{\mathrm{T}}\left(Z_{2}\right) \ln \left(\frac{4 \gamma_{1} \gamma_{2}^{2}}{E_{\gamma} R}\right) \frac{\mathrm{d} E_{\gamma}}{E_{\gamma}} .
$$

Analogously,

$$
\mathrm{d} \sigma_{\mathrm{br}}^{b}=\frac{2}{\pi} Z_{2}^{2} \alpha \sigma_{\mathrm{T}}\left(Z_{1}\right) \ln \left(\frac{4 \gamma_{2} \gamma_{1}^{2}}{E_{\gamma} R}\right) \frac{\mathrm{d} E_{\gamma}}{E_{\gamma}} .
$$

To compare these cross sections with the corresponding ones for the Delbrück scattering, we consider the case of identical nuclei and calculate the contribution of the ordinary nuclear bremsstrahlung integrated over the region $m<E_{\gamma}<m \gamma$, which is the main region for the total cross section $\sigma$ from (31). This gives

$$
\begin{aligned}
\Delta \sigma_{\mathrm{br}} & =\frac{2}{\pi} Z^{2} \alpha \sigma_{\mathrm{T}}(Z) L\left(L+l_{\gamma}\right), \\
l_{\gamma} & =\ln \left(\frac{4 \sqrt{\gamma}}{m R}\right) .
\end{aligned}
$$

As a result, we find that the ratio

$$
\frac{\Delta \sigma_{\mathrm{br}}}{\sigma}=\frac{\sigma_{\mathrm{T}}(Z)}{\sigma_{\mathrm{D}}(Z)}\left(1+\frac{l_{\gamma}}{L}\right)
$$

is indeed small:

$$
\frac{\Delta \sigma_{\mathrm{br}}}{\sigma} \approx \frac{1}{15} \text { for RHIC and } \approx \frac{1}{19} \text { for LHC } .
$$

\subsection{Comparison to $Z_{1} Z_{2} \rightarrow Z_{1} Z_{2} e^{+} e^{-\gamma}$}

An alternative source of photons in nuclear collisions is provided by the process

$$
Z_{1} Z_{2} \rightarrow Z_{1} Z_{2} e^{+} e^{-} \gamma
$$

in which the photon is emitted by an electron or a positron produced in collisions of the nuclei (Fig. 7). Such a process has been calculated in [14] using various methods for the numerical evaluation of its cross section. Strictly speaking, the final state of this process is different from that of $Z_{1} Z_{2} \rightarrow Z_{1} Z_{2} \gamma$. Nevertheless, it is of interest to compare the differential over the photon momentum distribution in 


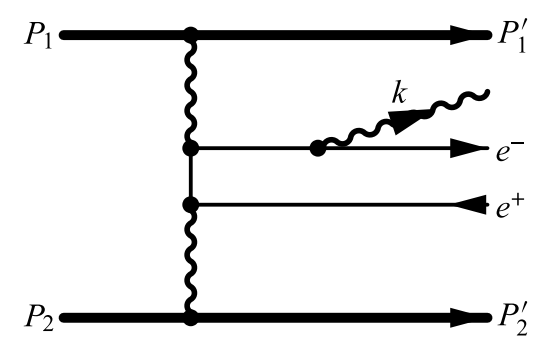

Fig. 7. Emission of photon by electron or positron, produced in a nuclear collision

this process with that given by (47). For this purpose, we use the analytic expression from [15] (derived originally for the $e^{+} e^{-}$collisions), which we denote by $\mathrm{d} \sigma_{\mathrm{FKh}}$, in order to reflect the names of the authors of the publication [15],

$$
\begin{aligned}
\mathrm{d} \sigma_{\mathrm{FKh}} & =\frac{7}{3 \pi^{3}} \frac{\alpha(Z \alpha)^{4}}{k_{\perp}^{4}} L^{2}\left(L_{0}-0.06\right) \frac{\mathrm{d}^{3} k}{E_{\gamma}}, \\
L_{0} & =\ln \left(\frac{k_{\perp}^{2}}{m^{2}}\right)
\end{aligned}
$$

which is valid for $k_{\perp}^{2} \gg m^{2}$. For $\mathrm{Au}-\mathrm{Au}$ collisions at the RHIC collider, the ratio

$$
\frac{\mathrm{d} \sigma_{\mathrm{Eq} .(47)}}{\mathrm{d} \sigma_{\mathrm{FKh}}}=\frac{6 \pi}{7} \frac{(Z \alpha)^{2} f_{Z}\left(k_{\perp} / m\right)}{L\left(L_{0}-0.06\right)} \approx \frac{1}{25},
$$

for $k_{\perp}^{2}=20 m^{2}$.

In other words, the mechanism of the associative production of photons as shown in Fig. 7 looks much more efficient than that considered in this paper. However, the estimate (62) is quite rough, since the analytic expression (61) is valid for large photon emission angles, but our equation (47) is correct for small emission angles. It should also be mentioned that numerical estimates in [14] give results that are by one order of magnitude smaller that those given by (61) (see, for example, Fig. 5 in [14]). It means that the yield of photons in the process in Fig. 7 exceeds that in the process considered not by far. Due to the difference of the final states in these processes, we can conclude that under a suitable differentiation of events by detectors, our virtual Delbrück scattering process can be observed if a reasonable efficiency of electron or positron recording can be achieved.

\section{Delbrück scattering and the $e Z \rightarrow e Z_{\gamma}$ process}

In this section we discuss briefly the process of a photon emission in electron-nucleus collisions without excitation of nucleus in the final state:

$$
e\left(P_{1}\right)+Z\left(P_{2}\right) \rightarrow e\left(P_{1}^{\prime}\right)+Z\left(P_{2}^{\prime}\right)+\gamma(k) .
$$

As was mentioned in Sect. 1, this consideration is motivated by a project of the eRHIC collider [8], with parameters

$$
Z_{1}=-1, \gamma_{1}=2 \times 10^{4}, Z_{2} \equiv Z=79, \gamma_{2}=108 .
$$

The process (63) via virtual Compton scattering and via virtual Delbrück scattering is described by the Feynman diagrams of Figs. 1 and 2, respectively, in which the first nucleus is replaced by the electron. The corresponding calculations are basically the same as above with some minor changes. In particular, in (17) we can neglect not only $\sigma_{2}$, but $\tilde{\sigma}_{1}$ as well:

$$
\mathrm{d} \sigma=\mathrm{d} \sigma_{1}=\mathrm{d} n_{e} \sigma_{\mathrm{D}}(Z)
$$

with (see, e.g., [13])

$$
\begin{aligned}
\mathrm{d} n_{e} & =2 \frac{\alpha}{\pi}\left(1-x+\frac{1}{2} x^{2}\right) \frac{\mathrm{d} \omega}{\omega} \ln \left(\frac{m \gamma_{1} \sqrt{1-x}}{\omega}\right), \\
x & =\frac{\omega}{E_{e}} .
\end{aligned}
$$

The total cross section is

$$
\sigma=\frac{\alpha}{\pi} \sigma_{\mathrm{D}}(Z) L^{2},
$$

which leads to the value

$$
\sigma=0.19 \times 10^{-3} \text { barn }
$$

for the parameters given in (64).

However, the main interest to this process is connected not with the total cross section, but with the spectrum of photons flying along the direction of the nuclear beam, i.e. in the subregion $A$ [see (39)]; this spectrum is given by

$$
\begin{aligned}
& \mathrm{d} \sigma^{(A)}=\frac{2 \alpha}{\pi} \sigma_{\mathrm{D}}(Z) \ln \frac{\gamma_{1} E_{\gamma}}{m} \frac{\mathrm{d} E_{\gamma}}{E_{\gamma}}, \\
& m \ll E_{\gamma} \ll m \gamma_{2} .
\end{aligned}
$$

By contrast, the spectrum of the ordinary nuclear bremsstrahlung in the same direction and the same region of energy has the form

$$
\mathrm{d} \sigma_{\mathrm{br}}^{a}=\frac{2 \alpha}{\pi} \sigma_{\mathrm{T}}(Z) \ln \left(\frac{4 \gamma_{1} \gamma_{2}^{2}}{E_{\gamma} R}\right) \frac{\mathrm{d} E_{\gamma}}{E_{\gamma}},
$$

and the integrated contribution of this spectrum from the photon energy region $m<E_{\gamma}<m \gamma_{2}$ is small,

$$
\frac{\Delta \sigma_{\mathrm{br}}^{a}}{\sigma^{(A)}} \approx \frac{1}{15}
$$

again for the eRHIC parameters given in (64).

In the subregion $B$ [see (40)], the photons fly along the electron beam direction, and their spectrum is

$$
\begin{aligned}
\mathrm{d} \sigma^{(B)}= & \frac{2 \alpha}{\pi} \sigma_{\mathrm{D}}(Z)\left(1-x_{\gamma}+\frac{1}{2} x_{\gamma}^{2}\right) \\
& \times \ln \left(\frac{m \gamma_{1} \sqrt{1-x_{\gamma}}}{E_{\gamma}}\right) \frac{\mathrm{d} E_{\gamma}}{E_{\gamma}},
\end{aligned}
$$

for $m \ll E_{\gamma} \ll m \gamma_{1}$, and we use the notation $x_{\gamma}=E_{\gamma} / E_{e}$. However, in this direction the ordinary bremsstrahlung is 
absolutely dominant, since its spectrum, given by the wellknown Bethe-Heitler formula (see, for example, [16]), is determined by the Compton scattering off the electron:

$$
\begin{aligned}
\mathrm{d} \sigma_{\mathrm{br}}^{b} & \approx \frac{16}{3} \frac{Z^{2} \alpha^{3}}{m^{2}}\left(1-x_{\gamma}+\frac{3}{4} x_{\gamma}^{2}\right) L_{\gamma} \frac{\mathrm{d} E_{\gamma}}{E_{\gamma}}, \\
L_{\gamma} & =\ln \frac{4 \gamma_{1}^{2} \gamma_{2}\left(1-x_{\gamma}\right)}{x_{\gamma}} .
\end{aligned}
$$

At the end of this section we consider, for completeness, the contribution of the virtual Delbrück scattering to the emission of photons in the collision of a high-energy muon with a heavy nucleus at rest. Let $\mu$ and $\gamma_{\mu}$ be the mass and the Lorentz factor of the muon, respectively. In this case, the emission of photons via virtual Delbrück scattering is described by the cross section

$$
\begin{aligned}
& \mathrm{d} \sigma=\frac{2 \alpha}{\pi} \sigma_{\mathrm{D}}(Z) \ln \left(\frac{m \gamma_{\mu}}{E_{\gamma}}\right) \frac{\mathrm{d} E_{\gamma}}{E_{\gamma}}, \\
& m \ll E_{\gamma} \ll m \gamma_{\mu},
\end{aligned}
$$

while the ordinary nuclear bremsstrahlung in the same region of energy has the form

$$
\mathrm{d} \sigma_{\mathrm{br}} \approx \frac{16}{3} \frac{Z^{2} \alpha^{3}}{\mu^{2}} \ln \left(\frac{\gamma_{\mu}^{2}}{R E_{\gamma}}\right) \frac{\mathrm{d} E_{\gamma}}{E_{\gamma}} .
$$

As a result, for muon-nucleus collisions, the emission of photons via virtual Delbrück scattering is small compared to the ordinary muon bremsstrahlung. E.g., even for a uranium nucleus, the ratio $\mathrm{d} \sigma / \mathrm{d} \sigma_{\mathrm{br}}$ is only about $2 \%$ for $\gamma_{\mu}>10^{3}$.

\section{Conclusions}

We have considered photon emission in collisions of ultrarelativistic heavy nuclei via the virtual Delbrück scattering subprocess. Although our analysis has been more general, for reasons of clarity, we focus on the case of a symmetric collision with $\gamma=\gamma_{1}=\gamma_{2}$ and $Z=Z_{1}=Z_{2}$ in this summary. The emitted photon energy region we consider is

$$
m \ll E_{\gamma} \ll m \gamma
$$

in the collider reference system. In the leading logarithmic approximation, the total photon emission cross section corresponding to this photon energy region is given by (31) as

$$
\sigma=\frac{2 \alpha}{\pi} Z^{2} \sigma_{\mathrm{D}}(Z)\left[\ln \left(\gamma^{2}\right)\right]^{2}
$$

where the quantity $\sigma_{\mathrm{D}}(Z)$ is related to the high-energy limit of the Delbrück scattering cross section according to (7). Note that the factor $r_{Z}$ in (7) takes care of the reduction of this cross section by the Coulomb corrections, which are of relative orders $\sim(Z \alpha)^{2 n}$, and which reduce the lowest-order result by almost a factor two. Equation (31), in a certain sense, constitutes the main result of this paper. Corrections to this result are of the order of $1 / \ln \left(\gamma^{2}\right) \approx 0.06$ for the $\mathrm{LHC} \mathrm{Pb}-\mathrm{Pb}$ option.

The energy and angular distribution of photons, emitted due to the Delbrück subprocess, is given by (47) in the leading logarithmic approximation, where we recall that the function $f_{Z}\left(k_{\perp} / m\right)$ is slowly varying in terms of $k_{\perp} / m$, and that numerical values of this function can be found in $[10,12]$.

The cross section due to the virtual electron loop as given in Fig. 2 is found to be considerably larger than that for the usual "tree-level" nuclear bremsstrahlung given in Fig. 1. The ratio of these cross sections can be found in (58), and a numerical result is given in (59). Figuratively speaking, we can say that the considered process depicted in Fig. 2 represents a QED radiative loop correction that is by one order of magnitude larger than the tree-level process of Fig. 1.

If our process can be detected experimentally, then one can effectively study Delbrück scattering in the range of initial photon energies in the rest frame of the colliding nucleus up to $\omega_{\mathrm{L}} \sim 2 m \gamma_{1} \gamma_{2}$, which is $10 \mathrm{GeV}$ for RHIC, $8 \mathrm{TeV}$ for LHC and $2 \mathrm{TeV}$ for eRHIC.

Acknowledgements. We are grateful to G. Baur, V. Fadin and A. Milstein for useful discussions. V.G.S. acknowledges the warm hospitality of the Institute of Theoretical Physics of Heidelberg University and support by the Gesellschaft für Schwerionenforschung (GSI Darmstadt). This work is partially supported by Russian Foundation for Basic Research (code 06-02-16064) and by the Fund of Russian Scientific Schools (code 5362.2006.2). U.D.J. acknowledges support by Deutsche Forschungsgemeinschaft (Heisenberg program).

\section{References}

1. G. Baur, K. Hencken, D. Trautmann, S. Sadovsky, Y. Kharlov, Phys. Rep. 364, 359 (2002)

2. W.-M. Yao et al., J. Phys. G 33, 1 (2006)

3. R. Engel, A. Schiller, V.G. Serbo, Part. Accel. 56, 1 (1996)

4. D. Trbojevic, D. Gasner, W. MacKay, G. McIntyre, S. Peggs, V. Serbo, G. Kotkin, Experimental set-up to measure coherent bremsstrahlung and beam profiles at RHIC (8th European Particle Accelerator Conference EPAC 2002, 3-7 June, 2002, Paris, p. 1986), see http://cern.ch/AccelConf/e02/PAPERS/THPRI076.pdf

5. C.A. Bertulani, G. Baur, Phys. Rep. 163, 299 (1988)

6. G. Baur, C.A. Bertulani, Z. Phys. A 330, 77 (1988)

7. I.F. Ginzburg, U.D. Jentschura, V.G. Serbo, manuscript in prepation (2007)

8. A. Deshpande, R. Milner, R. Venugopalan, W. Vogelsang, Ann. Rev. Nucl. Part. Sci. 55, 165 (2005) [hep-ph/0506148]

9. I.F. Ginzburg, U.D. Jentschura, V.G. Serbo, Phys. Lett. B 658, $125(2008)$

10. A.I. Milstein, M. Schumacher, Phys. Rep. 243, 183 (1994)

11. S.Z. Akhmadaliev et al., Phys. Rev. C 58, 2844 (1998)

12. H. Falkenberg et al., Atom. Data Nucl. Data 50, 1 (1992) 
13. V.M. Budnev, I.F. Ginzburg, G.V. Meledin, V.G. Serbo, Phys. Rep. C 15, 181 (1975)

14. K. Hencken, D. Trautmann, G. Baur, Phys. Rev. C 60, 034901 (1999) [nucl-th/9903019]
15. V.S. Fadin, V.A. Khoze, Sov. Phys. JETP 17, 313 $(1973)$

16. V.B. Berestetskii, E.M. Lifshitz, L.P. Pitaevskii, Quantum Electrodynamics (Pergamon Press, Oxford, 1982) 